\title{
Remembering individual perspectives and needs in differentiated HIV care strategies
}

\author{
Sheree R Schwartz, Stefan Baral
}

Key Populations Program, Center for Public Health and Human Rights, Department of Epidemiology, Johns Hopkins School of Public Health, Baltimore, Maryland, USA

\section{Correspondence to} Dr Sheree R Schwartz, Key Populations Program, Center for Public Health and Human Rights, Department of Epidemiology, Johns Hopkins School of Public Health, Baltimore, MD 21205, USA; sschwartz@jhu.edu

Received 27 November 2018 Accepted 27 November 2018 Published Online First 27 December 2018

\section{Linked}

- http://dx.doi.org/10.1136/ bmjqs-2017-007564

\section{Check for updates}

(C) Author(s) (or their employer(s)) 2019. No commercial re-use. See rights and permissions. Published by BMJ.

To cite: Schwartz SR, Baral S. BMJ Qual Saf

2019:28:257-259.
In 2018, the HIV pandemic response has transitioned from an emergency approach to one that will be implemented over several decades and likely for our lifetimes. An estimated 37 million people now live with HIV, of whom 21.5 million people are on antiretroviral therapy (ART) including an estimated 17.5 million people who have achieved viral suppression. ${ }^{1}$ Thus, an estimated 20 million people either require ART or improved regimens and/ or adherence interventions. ${ }^{1}$ Though HIV diagnostic and prevention strategies are improving, stigma, implementation challenges and late diagnoses resulted in 1.8 million people acquiring HIV, indicating that HIV incidence has only been slowly decreasing over the last several years. ${ }^{1}$ Thus, the numbers of people requiring HIV treatment are likely to continue to increase over the coming years.

Effective support for the lifelong treatment needs of an increasing number of people living with HIV despite decreasing levels of resources requires a better understanding of the specific individual needs of people living with HIV and development of cost-effective and adaptive approaches to address these needs. ${ }^{2}$ Differentiated care models have been promoted in sub-Saharan Africa recently to address the heterogeneity in the treatment needs among people living with $\mathrm{HIV}^{3}{ }^{4}$ Men and women, old and young, pregnant and non-pregnant and individuals from urban versus rural communities may respond differently to service delivery models. ${ }^{5-7}$ Further, given that HIV transmission risks are not spread evenly across populations anywhere in the world, engagement in care and adherence challenges are likely to differ across populations as well. ${ }^{8-12}$ Marginalised populations, such as pregnant women, adolescents, female sex workers, transgender populations, men who have sex with men and other key populations, may require different service delivery approaches and/or different intensities of care in response to their treatment needs. ${ }^{613-15}$

Despite the acknowledgement that individual people living with HIV and healthcare systems would benefit from tailored approaches to treatment, the vertical and siloed nature of the HIV response has often limited more holistic approaches to care which might better account for patient characteristics and preferences. ${ }^{16}$ Differentiated care models provide significant opportunities for patient-centred care, but to date have primarily been used as a mechanism for task-shifting, decongestion of clinics and decentralisation of care for those patients stable on ART. ${ }^{617-19}$ These approaches are important for expanding ART programmes given the growing clinic volumes, but may not go far enough in recognising diversity of patient preferences and treatment needs given the goals of achieving long-term patient retention on ART and sustained viral suppression. ${ }^{6} 19$

In this issue of BMJ Quality \& Safety, Tran et al explore patients' perspectives on solutions to address the burden of HIV treatment as well as expert opinions regarding the feasibility of implementing patients' ideas. ${ }^{20}$ These data are from Cote d'Ivoire. They comprise a cross-sectional qualitative sample of 326 ART-experienced patients recruited across three health facilities in Abidjan, the country's largest city (population approximately 4.5 million), as well as independent evaluations from a group of six stakeholders within the country.

Three thematic areas emerged regarding patients' perceived treatment burdens: financial constraints, time allocated to treatment and the perception that 
engagement in healthcare is a constant reminder of one's HIV status. Suggestions of how to address these burdens also centred around three areas, including improvements to their personal care, improvements within clinic management and organisation, and more general improvements to the health system overall. Commonly reported solutions to improve care were reduced pills burdens and/or other treatment changes, decreased frequency of visits, structural changes resulting in decreased wait times for clinically stable patients and more patient involvement in their healthcare, including more comprehensive information about HIV and treatment, as well as inclusion in decision-making around their care. Experts, consulted independently, were in consensus that nearly half of the suggested solutions to addressing patient treatment burdens were easy to implement and low cost.

This study contributes to the limited evidence base for the empirical evaluation of patient preferences for differentiated service delivery models. The available evidence from discrete choice experiments and from other qualitative work suggests that non-stigmatising and compassionate care may be more valued than distance from the clinics and wait times. ${ }^{21}$ Studies have found mixed preferences for clinic versus community-based service delivery. ${ }^{15} 2223$ More highquality study of patient preferences and satisfaction in the design of differentiated service delivery models focused on people living with HIV across sub-Saharan Africa remains much needed.

Evaluation of differences in patient preferences for service delivery approaches as well as heterogeneity of patient outcomes will be essential if cost-effective solutions to differentiated service delivery are to be found. Pragmatic trials, adaptive interventions and other implementation science models tested in larger populations which embrace and address these differences may help to further refine scale-up approaches. Considerations of different individual and population needs may also offer answers as to why generic community-based test and treat models have not been successful at having a population impact that bends the curve of the HIV pandemic. ${ }^{2425}$ Furthermore, interventions considering not only structural changes to service delivery, but quality of patient care and support, issues highlighted by Tran et al, should be assessed for comparative effectiveness with feasibility in mind. ${ }^{20}$ Effective, but more resource-intensive elements may still be appropriate for scale-up, but within specific subsets of populations or among treatment non-responders.

HIV remains one of the fundamental infectious disease pandemics in recorded history. Advancing the effectiveness of the health system response to HIV requires integration of patient preferences in programmes and the identification of who requires more intensive services and who would benefit from less onerous treatment support. ${ }^{19}$ To achieve sustained treatment goals across populations, lighter-touch models including less frequent visit attendance, adherence clubs, peer or lay healthcare workers, fast-track visits may be viable solutions for many clinically stable patients, but will likely need to be accompanied by more resource-intensive strategies for patients more vulnerable to being lost to care. ${ }^{26}$ Finally, adaptive intervention approaches tend to be those that begin with the current standard of care or a single intervention and then iteratively add complementary interventions or increase the intensity of services for individuals that remain unresponsive. We are in the early days of characterising the relative utility of adaptive interventions, but conceptually this may represent an effective differentiated care approach that would respond to individual needs while allocating resources to those most in need and likely to benefit from additional support. ${ }^{27}$

Addressing the heterogeneity in HIV treatment needs and preferences across and within populations also means challenging assumptions of which unmet needs are contributing to epidemics across sub-Saharan Africa. ${ }^{12}$ Tran and colleagues provide a useful methodological approach and insights to start this conversation. However, increasing treatment needs and decreasing investments create urgency. Achieving an AIDS-free generation may remain out of sight at present, but we can certainly close the treatment gap and provide better services to those living with HIV in Cote D'Ivoire, across sub-Saharan Africa and the world more broadly. Progress towards this important goal will require more studies of this type of individual preferences and needs combined with tailored strategies to address them.

Funding SS and SB were supported by the National Institutes of Health (NINR R01NR016650) as well as through support from the Johns Hopkins University Center for AIDS Research through the National Institutes of Health (award P30AI094189). The content is solely the responsibility of the authors and does not necessarily represent the official views of the National Institutes of Health.

Competing interests None declared.

Patient consent Not required.

Provenance and peer review Commissioned; internally peer reviewed.

\section{REFERENCES}

1 UNAIDS. UNAIDS fact sheet - July 2018. New York, NY: UNAIDS, 2008.

2 Kates J, Wexler A, Lief E. Donor government funding for HIV in low-and middle-income countries in 2016. Menlo Park, CA: The Henry J Kaiser Family Foundation \& UNAIDS, 2017.

3 International AIDS Society. Differentiated care for HIV: a decision framework for antiretroviral therapy delivery. Geneva: International AIDS Society, 2016.

4 Grimsrud A, Bygrave H, Doherty M, et al. Reimagining HIV service delivery: the role of differentiated care from prevention to suppression. J Int AIDS Soc 2016;19:21484. 
5 Murray KR, Dulli LS, Ridgeway K, et al. Improving retention in HIV care among adolescents and adults in low- and middleincome countries: A systematic review of the literature. PLoS One 2017;12:e184879.

6 World Health Organization. Key considerations for differentiated antiretroviral therapy delivery for specific populations: children, adolescents, pregnant and breastfeeding women and key populations. Geneva: World Health Organization, 2017.

7 Hodgson I, Plummer ML, Konopka SN, et al. A systematic review of individual and contextual factors affecting ART initiation, adherence, and retention for HIV-infected pregnant and postpartum women. PLoS One 2014;9:e111421.

8 Baral S, Beyrer C, Muessig K, et al. Burden of HIV among female sex workers in low-income and middle-income countries: a systematic review and meta-analysis. Lancet Infect Dis 2012;12:538-49.

9 Baral S, Sifakis F, Cleghorn F, et al. Elevated risk for HIV infection among men who have sex with men in low- and middle-income countries 2000-2006: a systematic review. PLoS Med 2007;4:e339.

10 Baral SD, Grosso A, Holland C, et al. The epidemiology of HIV among men who have sex with men in countries with generalized HIV epidemics. Curr Opin HIV AIDS 2014;9:156-67.

11 Beyrer C, Baral SD, van Griensven F, et al. Global epidemiology of HIV infection in men who have sex with men. The Lancet 2012;380:367-77.

12 Baral S, Phaswana-Mafuya N. Rewriting the narrative of the epidemiology of HIV in sub-Saharan Africa. SAHARA-J: Journal of Social Aspects of HIV/AIDS 2012;9:127-30.

13 Macdonald V, Verster A, Baggaley R. A call for differentiated approaches to delivering HIV services to key populations. J Int AIDS Soc 2017;20:21658.

14 Clouse K, Motlhatlhedi M, Bonnet K, et al. "I just wish that everything is in one place": facilitators and barriers to continuity of care among HIV-positive, postpartum women with a non-communicable disease in South Africa. AIDS Care 2018;30(sup2):5-10.

15 Myer L, Iyun V, Zerbe A, et al. Differentiated models of care for postpartum women on antiretroviral therapy in Cape Town, South Africa: a cohort study. J Int AIDS Soc 2017;20:21636.

16 Mounier-Jack S, Mayhew SH, Mays N. Integrated care: learning between high-income, and low- and middle-income country health systems. Health Policy Plan 2017;32(suppl_4):i v6-iv12.
17 Republic of South Africa National Department of Health. Circular on the implementation of the universal test and treat strategy for hiv positive patients and differentiated care for stable patients. Pretoria, South Africa: Republic of South Africa National Department of Health, 2016.

18 Waldrop G, Doherty M, Vitoria M, et al. Stable patients and patients with advanced disease: consensus definitions to support sustained scale up of antiretroviral therapy. Trop Med Int Health 2016;21:1124-30.

19 Grimsrud A, Barnabas RV, Ehrenkranz P, et al. Evidence for scale up: the differentiated care research agenda. J Int AIDS Soc 2017;20(Suppl 4):22024.

20 Tran V-T, Messou E, Mama Djima M, et al. Patients' perspectives on how to decrease the burden of treatment: a qualitative study of HIV care in sub-Saharan Africa. BMJ Qual Saf 2019;28:266-75.

21 Zanolini A, Sikombe K, Sikazwe I, et al. Understanding preferences for HIV care and treatment in Zambia: Evidence from a discrete choice experiment among patients who have been lost to follow-up. PLoS Med 2018;15:e1002636.

22 Hanrahan C, Soyizwaphi P, Keyser V. Acceptability of community and clinic-based adherence clubs for stable-ART patients.. Presented as an oral presentation at the Conference of the South African HIV Clinicians Society, Johannesburg, South Africa, 2016.

23 Comins C, Parmley L, Schwartz S. Barriers and facilitators to successful decentralized treatment provision for female sex workers living with HIV in Durban, South Africa. Presented as an oral poster at the International AIDS Conference, Amsterdam, The Netherlands, 2018.

24 Iwuji CC, Orne-Gliemann J, Larmarange J, et al. Universal test and treat and the HIV epidemic in rural South Africa: a phase 4, open-label, community cluster randomised trial. Lancet HIV 2018;5:e116-e125.

25 Havlir D, Charlebois E, Balzer L. SEARCH community cluster randomized study of HIV "test and treat" using multi- disease approach and streamlined care in rural Uganda and Kenya. Presented as an oral presentation at the International AIDS Conference, Amsterdam, The Netherlands, 2018.

26 Barker C, Dutta A, Klein K. Can differentiated care models solve the crisis in HIV treatment financing? Analysis of prospects for 38 countries in sub-Saharan Africa. J Int AIDS Soc 2017;20:21648.

27 Kidwell KM, Hyde LW. Adaptive Interventions and SMART Designs: application to child behavior research in a community setting. Am J Eval 2016;37:344-63. 